\title{
Duals of Banach spaces which admit nontrivial smooth functions
}

\section{K. John and V. Zizler}

If a Banach space $X$ admits a continuously Fréchet differentiable function with bounded nonempty support, then $X^{*}$ admits a projectional resolution of identity and a continuous linear one-to-one map into $c_{0}(\Gamma)$.

\section{Introduction}

There are two difficulties in building up the projectional resolution of identity in nonseparable Banach spaces; such a resolution was originally constructed by Amir and Lindenstrauss ([1]) for spaces which are generated by a weakly compact set. First we need a compactness argument to ensure the existence of limit points for certain nets of operators and second we need to be able to ensure that the limit point is a projection. The first one can be overcome in any dual space. Tacon showed in ([4]) that also the second difficulty can be overcome in duals of spaces with Fréchet smooth norm. His argument relies on the uniqueness of Hahn-Banach extensions. Here we show that the projectional resolution of identity in $X^{*}$ exists under the hypotheses in the abstract. This is done by basing the proof on the existence of differentials of certain functions constructed by Leduc ([2], [3]).

\section{Notations and definitions}

We will work in real Banach spaces. The norm $|\cdot|$ of a Banach space $X$ is rotund if whenever $|x+y|=2,|x|=|y|=1$, then $x=y$. If $X$

Received 17 April 1974. 
is a Banach space, then, following [4], $X^{\alpha}$ is the Banach space of all bounded homogeneous functionals on $X$ with the sup-norm over the unit ball of $X$. If $C$ is a subspace of $X$ and $T: C^{*} \rightarrow X^{*}$ is a bounded linear map, then $\tilde{T}: X^{*} \rightarrow X^{*}$ is defined as $\tilde{T} f=T R f$, where $R$ means the restriction-map to $C^{*}$. dens $X$ is the smallest cardinality of a dense subset of a Banach space $X$. The symbol clM denotes the norm closure of $M$ in $X$.

\section{Main result}

THEOREM 1. Let $X$ be a Banach space which admits a continuously Fréchet differentiable function with bounded nonempty support. Let $\mu$ be the first ordinal of cardinality dens $X$. Then for every $0 \leq \alpha \leq \mu$ there is a subspace $X_{\alpha}$ of $X$ and a linear operator $T: X_{\alpha}^{*} \rightarrow X^{*}$ such that $P=\tilde{T}_{\alpha}$ is a linear projection with $X_{\alpha} \subset X_{\beta}$ if $\alpha<\beta$ and $X_{\mu}=X$, and

1. $\left|P_{\alpha}\right|=1$ for $\alpha>0, P_{0}=0$,

2. $P_{\alpha} X^{*}$ is linearly isometric to $X_{\alpha}^{*}, \operatorname{dens} X_{\alpha}\left(=\operatorname{dens} X_{\alpha}^{*}\right) \leq \overline{\bar{\alpha}}$ for infinite $\alpha$,

3. $P_{\alpha} P_{\beta}=P_{\beta} P_{\alpha}=P_{\beta}$, where $\beta<\alpha$,

4. $\underset{\beta<\gamma}{\bigcup} P_{B+1} X^{*}$ is norm dense in $P_{\gamma} X^{*}$, or equivalently

5. for every $x^{*} \in X^{*}, P_{\alpha^{x}}$ is norm continuous on ordinals.

COROLLARY. If a Banach space $X$ admits a continuously Fréchet differentiable function with bounded nonempty support, then $X^{*}$ admits a bounded linear one-to-one map into $c_{0}(\Gamma)$. Thus $X^{*}$ has an equivalent rotiond norm.

\section{Proof of the main result}

We need the following result of Amir and Lindenstrauss.

LEMMA 1 (see [1], Lemma 2). Asszome $X$ is a normed linear space. Then given $\varepsilon>0$, an integer $n>0, m$ elements $f_{1}, \ldots, f_{m}$ of $X^{*}$ and any finite dimensional subspace $B \subset X$, there is an $\kappa_{0}$-dimensional 
subspace $C \subset X$ containing $B$ such that, for every subspace $Z$ of $X$ with $Z \supset B$ and $\operatorname{dim} Z / B=n$, there is a linear operator $T: Z \rightarrow C$ with $|T| \leq 1+\varepsilon, T b=b$ for every $b \in B$ and $\left|f_{k}(z)-f_{k}(T z)\right| \leq \varepsilon|z|$ for every $z \in Z$ and $k=1,2, \ldots, m$.

Also we need the following result of Leduc.

LEMMA 2 (see [2], Theorem 3 and [3], Corollary 1). If $f$ is a continuously Fréchet differentiable real valued function on a Banach space $X$ with bounded nonempty support (we may assume $f(0)>0$ and $0 \leq f \leq 1$ ), then the gauge of $f$ defined by the formula

$$
v(x)=\left(\int_{-\infty}^{+\infty} f(t x) d t\right)^{-1}, x \neq 0,
$$

is continuously Eréchet differentiable, $\nu^{\prime}(x) \neq 0$ and

$$
c l\left\{\nu^{\prime}(x) \cdot\left|\nu^{\prime}(x)\right|^{-1},|x|=1\right\}=\left\{f \in X^{*},|f|=1\right\} \text {. }
$$

LEMMA 3. Let $X$ be a Banach space, $B$ a finite dimensional subspace of $X, f_{1}, \ldots, f_{m} \in X^{*}$. Then there is a separable subspace $C$ of $X$ and a linear operator $T: C^{*}+X^{*}$ such that $|T|=1$ and $\tilde{T}^{*} x=x$ for all $x \in B, \quad \tilde{T} f_{i}=f_{i}, \quad i=1,2, \ldots, m$.

Proof. Let $C_{n} \supset B, n=1,2, \ldots$, be the ${ }_{0}$-dimensional subspaces of $X$ given by Lemna I for $\varepsilon=1 / n$, and let $C=\overrightarrow{\mathrm{sp}}\left(\begin{array}{ll}\cup & C_{n} \\ n\end{array}\right)$. If $E$ is a subspace of $X, E \supset B, \operatorname{dim} E / B=n$, then there is a linear operator $T_{E}: E \rightarrow C$ such that $\left|T_{E}\right| \leq 1+1 / n, T_{E} x=x$ for $x \in B$, $\left|f_{k}\left(T_{E}\right)-f_{k}(z)\right| \leq \varepsilon|z|, z \in E, k=1,2, \ldots, m$. We extend $T_{E}$ to a homogeneous map $T_{E}^{\prime}: X \rightarrow C$ by $T_{E}^{\prime} x=0$ if $x \in X \backslash E$. We consider $T^{\prime *}: C^{*} \rightarrow X^{\alpha}$ where in the space of bounded linear maps $C^{*} \rightarrow X^{\alpha}$ we consider the pointwise topology and on $x^{\alpha}$ the $X$-topology. By the Tychonoff Theorem, the net $T_{E}^{\prime}$ has a limit point $T: C^{*} \rightarrow X^{*}$ and if $x \in X$, then

$$
\begin{aligned}
\left(\tilde{T} f_{j}\right)(x)=(T R) f_{j}(x) & = \\
& =\lim \left(T_{E}^{\prime} R f_{j}\right)(x)=\lim \left(T_{E}^{*} R f_{j}\right)(x)=\lim \left(R f_{j}\right) T_{E} x=f_{j}(x) .
\end{aligned}
$$


Similarly $\tilde{T}^{*} x=x$ for $x \in B$.

LEMMA 4. Let $X$ be a Banach space, $f$ a continuously Fréchet differentiable function on $X$ with bounded support such that $0 \leq f \leq 1$ and $f(0)>0$. Let $v$ be the gauge of $f$ defined in Lemma $2, \aleph$ an infinite cardinal number. Assume $2, W$ are subspaces of $X, X^{*}$ respectively, dens $Z$, dens $W \leq K$. Then there is a subspace. $C \subset X$, dens $C \leq \kappa, C \supset Z$, and a linear operator $T: C^{*} \rightarrow X^{*}$ with $|T|=I$, $T R g=g$ for $g \in W, \quad T R d=d$ for all differentials $d$ of $\nu$ at all points of $C \backslash\{0\}$ and $(T R)^{*} x=x$ for $x \in C$ and such that $T C^{*}=c l\{\lambda d, \lambda \geq 0, d$ differentials of $\nu$ at all points of $C \backslash\{0\}\}$. Then $P=T R$ is a projection on $X^{*}, \quad|P|=1$ such that $P g=g$ for $g \in W, P^{*} x=x$ for $x \in C$. Furthermore, $R: P X^{*} \rightarrow C^{*}$ is an isometry onto $C^{*}$.

Proof. By transfinite induction on $\kappa$. If $\kappa=\kappa_{0}$ and $x_{j}, f_{j}$, $j=1,2, \ldots$, are dense in $z, W$ respectively, then there exist, by Lemma 3 , separable subspaces $C_{n} \subset X, n=1,2, \ldots$, and linear operators $T_{n}: C_{n}^{*} \rightarrow X^{*}$ with $\left|T_{n}\right|=1, \tilde{T}_{n}^{*} x_{i}=x_{i}, i=1,2, \ldots, n$, and $\tilde{T}_{n}^{*} x_{i}^{k}=x_{i}^{k}, 1 \leq i \leq n, 1 \leq k \leq n-1$, where $0 \neq x_{i}^{k}$, $i=1,2, \ldots$, is dense in $C_{k}, T_{n} f_{i}=f_{i}, i=1,2, \ldots, n, T_{n} d=d$ for all differentials $d$ of $v$ at $x_{i}^{k}, 1 \leq i \leq n, 1 \leq k \leq n-1$. Let us put $C=\operatorname{cl} \bigcup_{n} C_{n}$. If $R_{n}$ is the restriction map of $C^{*}$ to $C_{n}^{*}$, then the limit point $T$ in the $X$-operator topology of the net $\left\{T_{n}{ }_{n}\right\}_{n}$ is seen by the arguments used in Lemma 3 to satisfy that if $P=\tilde{T}$, then $|P|=1, P$ is linear, $P^{*} x_{i}^{k}=x_{i}^{k}$ for $i, k=1,2, \ldots$, so that $P^{*} x=x$ for all $x \in C$ and similarly $P f=f$ for all $f \in W, P d=d$ for all differentials $d$ of $v$ at all $x \in C, x \neq 0$. Here we use the continuous Fréchet differentiability of $v$ on $X \backslash\{0\}$. It remains to prove that $P$ is a projection; that is, $P^{2}=P$. To show this it clearly suffices to prove that $P X=c l\{\lambda d, d$ differential of $\nu$ at a nonzero point of $c, \lambda \geq 0\} \equiv D$. 
If $d \in D$, then for some sequence $\lambda_{i} \geq 0, d_{i}$ differentials of $v$ at $c \backslash\{0\}, \lim \lambda_{i} d_{i}=d$. Then $P d=P\left(\lim \lambda_{i} d_{i}\right)=\lim \lambda_{i} d_{i}=d$, so $D \subset P X^{*}$. If $x^{*}=T C^{*}, c^{*} \in C^{*}$, then Lemma 2 used for $C$ gives the existence of differentials $d_{i}$ of $v$ at the points of $C \backslash\{0\}$ and $\lambda_{i} \geq 0$ such that $\lim \lambda_{i} R d_{i}=e^{*}$, where $R d_{i}$ is the restriction of $d_{i}$ to $C$. So, $T C^{*}=T\left(\lim \lambda_{i} R d_{i}\right)=\lim T R\left(\lambda_{i} d_{i}\right)=\lim \lambda_{i} d_{i}$, showing that $P X^{*} \subset D$.

Now we show that the restriction $R: P X^{*} \rightarrow C^{*}$ is an isometry onto. For if $c^{*} \in C,\left|c^{*}\right|=1, \varepsilon>0$, then there is a $c \in C,|c|=1$, such that $\left|c^{*}(c)-1\right|<\varepsilon$. So if $x^{*} \in X^{*}$, $c^{*}=R x^{*}$, then $P x^{*}=T c^{*}$ and $\left(P x^{*}\right)(c)=c^{*}\left(P^{*} c\right)=c^{*}(c)$. From the last fact and from $|P|=1$ easily follows that $R$ is an isometry. Furthermore $R P X^{*} \supset R D$, so $R$ is onto $C^{*}$ by use of Lemma 2. If the lemma holds for all cardinals less than $\aleph$ and $\mu$ is the first ordinal of $\overline{\bar{\mu}}=\aleph$, then obviously there are suospaces $z_{\alpha} \subset Z, W_{\alpha} \subset W, \alpha<\mu$ such that $z_{\alpha} \subset z_{\beta}, W_{\alpha} \subset W_{\beta}$ if $\alpha<\beta$ with $\operatorname{dens} Z_{\alpha}$, dens $W_{\alpha} \leq \overline{\bar{\alpha}}$ and $z=c l \underset{\alpha<\mu}{\cup} z_{\alpha}, W=c l \underset{\alpha<\mu}{U} W_{\alpha} \cdot$ By the induction hypothesis, we construct for every $\alpha<\mu$, a subspace $C_{\alpha} \subset X$ with dens $C_{\alpha} \leq \overline{\bar{\alpha}}$ and such that $C_{\alpha} \supset z_{\alpha} \cup \underset{\beta<\alpha}{\cup} C_{\beta}$ together with a linear operator $T_{\alpha}: C_{\alpha}^{*} \rightarrow X^{*}$ such that $P=\tilde{T}_{\alpha}$ satisfies $\left|P_{\alpha}\right|=1$, $P_{\alpha}^{*} x=x$ for $x \in C_{\alpha}, P_{\alpha} f=f$ for $f \in W_{\alpha}$, $P_{\alpha} X^{*}=\operatorname{cl}\left\{\lambda d, \lambda \geq 0, d\right.$ differentials of $\nu$ at the points of $\left.C_{\alpha} \backslash\{0\}\right\}$. We put $C=c l \underset{\alpha<\mu}{\bigcup} C_{\alpha}$ and consider the extensions of $T_{\alpha}, \tilde{T}_{\alpha}: C^{*} \rightarrow X^{*}$. Again for $T$ we take a limit point in the $X$-operator topology of $\tilde{T}_{\alpha}$, $\alpha<\mu$ and see that

$T X^{*}=\{\lambda d, d$ differentials of $\nu$ at nonzero points of $c, \lambda \geq 0\}$ and $P=\tilde{T}$ satisfies our requirements.

Proof of Theorem. From Lemma 5 and the arguments developed in [1], [4], the theorem follows.

Proof of Corollary. It is the same as the proof of Theorem 1 and its corollary in [4]. 


\section{References}

[1] D. Amir and J. Lindenstrauss, "The structure of weakly compact sets in Banach spaces", Ann. of Math. (2) 88 (1968), 35-46.

[2] Michel Leduc, "Jauges différentiables et partitions de l'unité", Séminaire Choquet, Initiation à l'analyse, 4e année, 1964/65, 12-01 - 12-10 (Mathematica Seminosa, 1. Secrétariat Mathématique, Paris, 1965).

[3] Michel Leduc, "Densité de certaines familles d'hyperplans tangents", C.R. Acad. Sci. Paris Sér. A 270 (1970), 326-328.

[4] D.C. Tacon, "The conjugate of a smooth Banach space", BuZZ. Austral. Math. Soc. 2 (1970), 415-425.

Mathematical Institute,

Czechoslovak Academy of Sciences,

Praha;

Department of Mathematics,

Charles University,

Praha,

Czechoslovakia. 